\title{
Lessons learned with the Active Phasing Experiment: comparison of four optical phasing sensors on a segmented Very Large Telescope
}

\author{
F. Gonte ${ }^{1, a}$ I. Surdej ${ }^{1}$ \\ ${ }^{1}$ European Southern Observatory, Garching, Germany
}

\begin{abstract}
The adaptive optics capabilities are strongly limited by the quality of the phasing of the primary mirror of the extremely large telescope. Up to date, the Keck telescopes are the only segmented telescope phased with a quality enabling the application of adaptive optics. The Active Phasing Experiment has been installed at the Namyth focus of the Very Large Telescope Melipal during the last 6 months. Its purpose is to understand and compare different technological concepts for an optical phasing sensor dedicated to the European Extremely Large Telescope. The pupil of the telescope is segmented in 61 hexagonal segments by projecting it on an Active Segmented Mirror. The ASM is controlled by a dual wavenlength interferometer made by Fogale Nanotech with a nanometric precision. The segmented pupil is distributed in parallel to four optical phasing sensors. They are a pyramid sensor, a curvature sensor, a phase filtering sensor and a ShackHartmann sensor. They have been developed respectively by Istituto Nazionale di Astrofisica in Florenze, Instituto Astrofisica Canarias in Tenerife, Laboratoire d'Astrophysique de Marseille and ESO. The global behaviour of the optical phasing sensors will be described and preliminary results of the Active Phasing Experiments obtained on sky will be explained. The extrapolation of the results to the EELT and the potential consequences for the adaptive optics will be given. The Active Phasing Experiment has been financed by the European Union and the European Southern Observatory via the Sixth European Union Framework Program for Research and Technological Development under the contract number 011863.
\end{abstract}

\section{Introduction}

Some of the next generation of giant optical telescopes will be equipped with segmented primary mirrors composed of hundreds of hexagonal segments. It is necessary to operate at the diffraction limit of such telescopes if the telescope is to use adaptive optics and be a science driver, and this can only be achieved if the segments are well-aligned both in height, called from now on "piston", and in tip and tilt. The fast control of the rigid body positions of the segments will be based on measurements made with edge sensors. These, however, can only measure differential movements between adjacent segments and therefore have to be supplied with reference values for the absolute measurements of the piston steps at the intra-segment borders. At the moment, such reference values can only be obtained with a precision of the order of a few nanometres by optical measurements, preferably using the light of a star in the field of the telescope.

The Active Phasing Experiment has then been proposed by the ESO to demonstrate its capability to align in tip-tilt and piston within few nanometres the segmented primary mirror of a giant telescope, performance which has not been made up to now in Europe. The goal was to simulate a VLT having

\footnotetext{
a e-mail : fgonte@eso.org
} 
a segmented primary mirror composed of 61 segments in order to demonstrate the functionality of 4 different optical phasing technologies and also new control principles using theses Optical Phasing Sensors (OPS).

\section{APE Design}

APE has been designed as a modular system on a $3 \mathrm{~m}$ by $2 \mathrm{~m}$ optical bench. The main subsystems are a turbulence generator (called MAPS), the Active Segmented Mirror, the Internal Metrology, four optical phasing sensors and the junction boxes. Three electronics cabinets contain the amplifiers, the analogue-to-digital converter cards, the controllers and the nine central processing units required for the control of the electronic components of APE (six CCDs, five translation stages, twelve rotating stages, two fast steering mirrors and the 183 actuators of the 61 segments incorporated in the ASM). The cabinets are linked to the bench via the junction boxes.

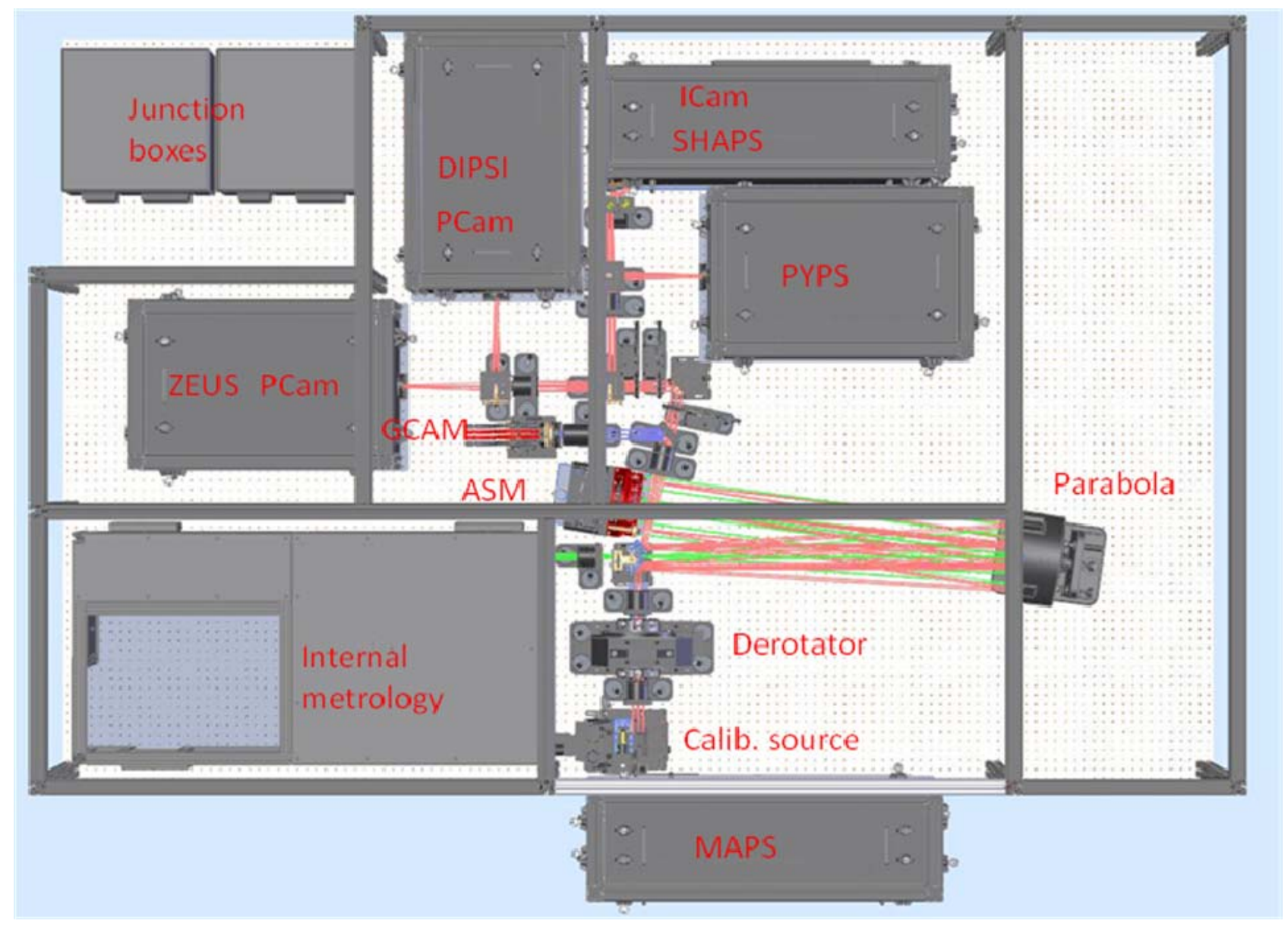

Fig. 1. Top view of the APE bench with its main subsystems in laboratory.

Figure 1 shows a top view of APE with its two independent optical paths. The path in pink that includes a reflection of the ASM, feeds the OPSs, with the light coming from either the telescope focus or from MAPS. The path shown in green is the one used by the IM to measure the position of the ASM segments. In total there are 42 optical surfaces (lenses, filters, mirrors, beam splitters, etc.) between the focus of the telescope and the entrance foci of the phasing sensors. The quality of the wavefront delivered to the phasing sensors is better than $\lambda / 2$ peak-to-valley across the segmented pupil. APE operates at wavelengths between $500 \mathrm{~nm}$ and $920 \mathrm{~nm}$, with the bandpass between 820 $\mathrm{nm}$ and $880 \mathrm{~nm}$ reserved for the IM. The sensor units, fixed to the bench by kinematic interfaces, the optics and the other subsystems are aligned with a precision better than $100 \mu \mathrm{m}$. 


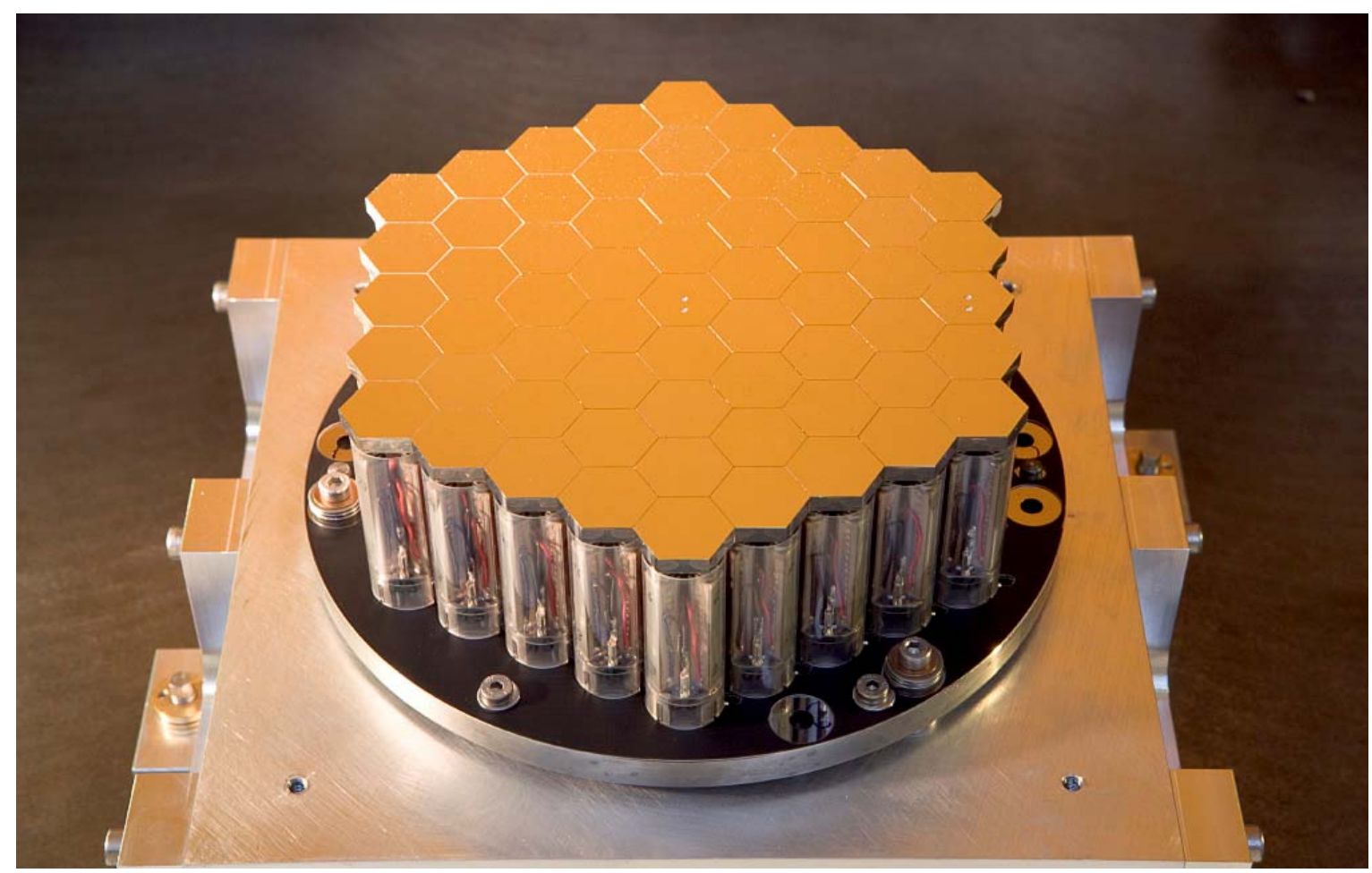

Fig. 2. Signal obtained by each optical phasing sensor.

The ASM, shown in Figure 2 before its installation on the bench, is a flat mirror composed of 61 hexagonal segments, made of Zerodur with a reflective coating of aluminium. The wavefront quality of the segments is better than $15 \mathrm{~nm}$ rms. They can be positioned in piston, tip and tilt by three piezo-actuators with strokes of $30 \mu \mathrm{m}$ and a resolution of $0.5 \mathrm{~nm}$. The segments have inner diameters of $17 \mathrm{~mm}$ with gaps between them of the order of $100 \mu \mathrm{m}$. The inner and outer diameters of the pupil of the ASM are $139 \mathrm{~mm}$ and $154 \mathrm{~mm}$, respectively. Projecting the segments onto the primary mirror of the VLT, the telescope with a meniscus primary mirror appears transformed into a segmented telescope with 61 segments of $1.05 \mathrm{~m}$ diameter, with gaps between the segments of $4 \mathrm{~mm}$ and with the capability of correcting piston, tip and tilt with a precision of $0.5 \mathrm{~nm}$. The heart of APE is the ASM, but its pacemaker is the IM developed by Fogale Nanotech [1]. It is a dual wavelength (835 and $860 \mathrm{~nm}$ ) phase shifting interferometer with two optical arms with lengths of $2.5 \mathrm{~m}$ each. The first arm located inside the IM unit serves as the reference. The second arm goes back and forth between the IM and the ASM via an off-axis parabola. The IM measures the position of each segment of the ASM with respect to the central segment at a frequency of $8 \mathrm{~Hz}$ with a precision better than $0.3 \mathrm{~nm}$. Like any other interferometer, the system is sensitive to environmental disturbances like vibrations of the bench and air turbulence due to temperature inhomogeneities or wind, all creating noise in the measurement and consequently a loss of resolution in the control loop between the ASM and the IM. In the laboratory the APE bench was placed on a pneumatic support to filter the vibrations coming from the ground. In addition, the temperature was controlled. The IM resolution obtained in the on the Nasmyth platform during observation for the positioning of the segments relative to the central one was better than $3 \mathrm{~nm}$ rms. Approximately $50 \%$ of the manpower dedicated to APE has been spent on the development of the VLT-compliant software, covering a variety of tasks ranging from the control of the motors to the archiving of the data gathered by APE and its phasing sensors. The complexity of the software is due, on the one hand, to the need for high flexibility in the configuration and execution of the experiment and, on the other hand, to the elaborate algorithms used for the analyses of the data delivered by the four phasing sensors. 


\section{The Four Optical Phasing Sensors}

Four different types of OPSs were built and tested. Figure 3 shows typical signals obtained by the four OPSs. DIPSI [2] is a curvature sensor analysing defocused images of the pupil. For low order wavefront errors the signal is approximately proportional to the second derivative of the wavefront and is easily interpreted using geometrical optical theory. For sharp discontinuities generated by phase steps at segment borders, the explanation of the signal requires Fresnel diffraction theory. The amplitude of the signal has been used as the estimator for the piston step. PYPS [3] is based on the pyramid sensor technology, originally developed for adaptive optics. PYPS produces an optical signal by splitting the image in a focal plane by a pyramid with four faces. This is equivalent to a simultaneous knife-edge test on two orthogonal axes. The signal is constructed from the intensities in the four pupil images and, broadly speaking, is proportional to the first derivative of the local wavefront. A simulated or measured interaction matrix is then used to retrieve the piston, tip and tilt errors of each segment. To determine the interaction matrix a record of the signal for each degree of freedom of the segmented mirror is made and the pseudo-inverse of the matrix is generated. The interaction matrix depends on the number of modes to be corrected. This approach has the advantage that it captures all parameters that affect the signal. The disadvantage is that interaction matrices have to be determined for a variety of parameters like the seeing conditions and the number of modes to be corrected. The modified feature of PYPS, compared to other pyramid sensors, such as the one used in the Multi-conjugate Adaptive Optics Demonstrator MAD [4], is that the focus on the pyramid is modulated by a fast steering mirror to increase the sensitivity of the sensor.
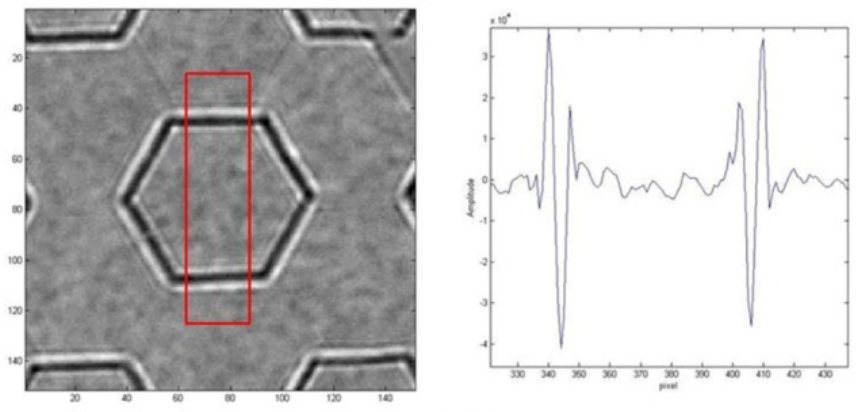

DIPSI
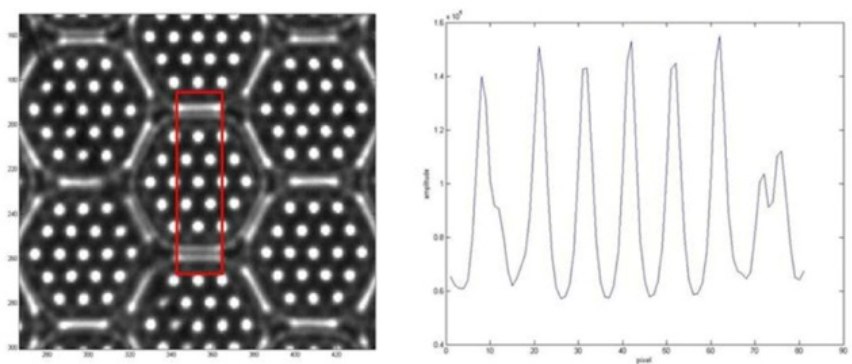

SHAPS
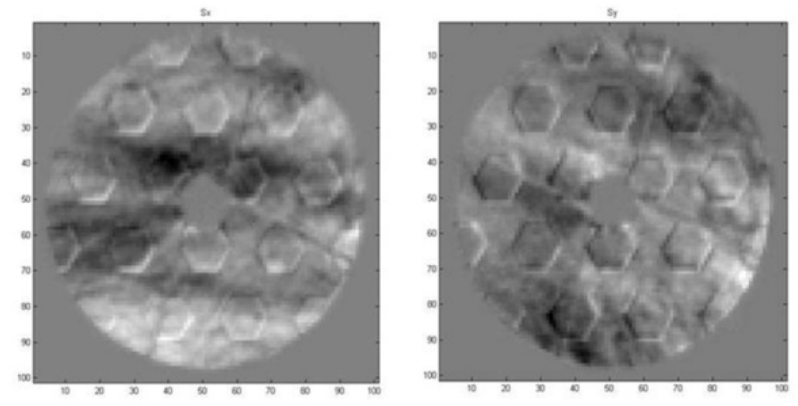

PYPS
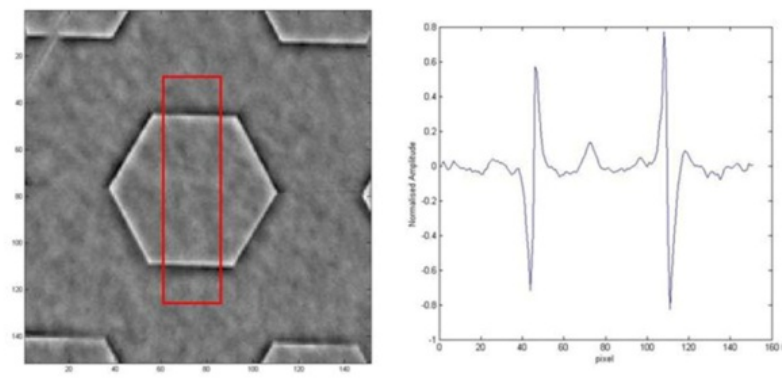

ZEUS

Fig. 3. Signal obtained by each optical phasing sensor.

SHAPS [5] is based on the Shack-Hartmann technology that has been used in active and adaptive optics for a long time. It is also the standard method used on the Keck telescopes to measure the positions of the segments. The lenslets are aligned so that the centres of the corresponding subapertures lie on the borders between two segments. The signals are the diffraction patterns of the lenslets. Although the piston step affects the position as well as the shape of the signal, only the shape is used as an estimator for the piston steps. SHAPS uses cylindrical lenslets covering the full border between two segments that provides the flexibility to average the signal along any fraction of the border and therefore measure the piston step along the whole border. The lenslet array also 
supplies 19 lenslets inside each segment subaperture for the measurement of the segment aberrations. ZEUS [6] is based on the modified Mach-Zehnder interferometer phasing sensor developed at LAM [7]. A phase and spatial filter is installed at the focus of the telescope to filter out low spatial frequencies in the wavefront, such as the ones generated by the atmosphere, thereby increasing the contrast for high frequency errors such as those due to segment piston errors. The optimum size of the mask is approximately equal to the diameter of the seeing disc. However, three different masks are sufficient to cover seeing conditions ranging from 0.4 to 2 arcsec. The normalised signal is constructed from three images: a signal taken with the filter; an image of the pupil taken without the filter; and a dark image. The piston steps are then estimated by fitting a theoretical expression to the signal. A simpler, but less accurate, method is to use only the amplitude as the estimator for the piston step.

All OPSs suffer from the same limitation. Using monochromatic light with a wavelength $\lambda$, the range of the measurable phase difference is limited to $(-\lambda / 2, \lambda / 2)$ and the signals are identical for piston steps differing by integral multiples of $\lambda$. The ambiguity can be resolved, and the capture range increased, if multiple measurements are performed with a small set of narrowband optical filters. This method, conventionally called the multi- $\lambda$ technique, allows a capture range of the order of a few microns, following which only a small number of iterations are necessary in closed loop to reduce the piston steps to values well within the $(-\lambda / 2, \lambda / 2)$ range. Another approach to resolve the ambiguity, usually called the coherence method, is to move adjacent segments with respect to each other over a large range of piston values. Using a broadband filter a signal will only appear if the piston step is sufficiently small. In principle, for all sensors, the highest precision will be obtained with narrowband optical filter measurements, provided that the sources are sufficiently bright. However, sufficient precision with faint stars can only be achieved with broadband optical filter measurements.

\section{Results}

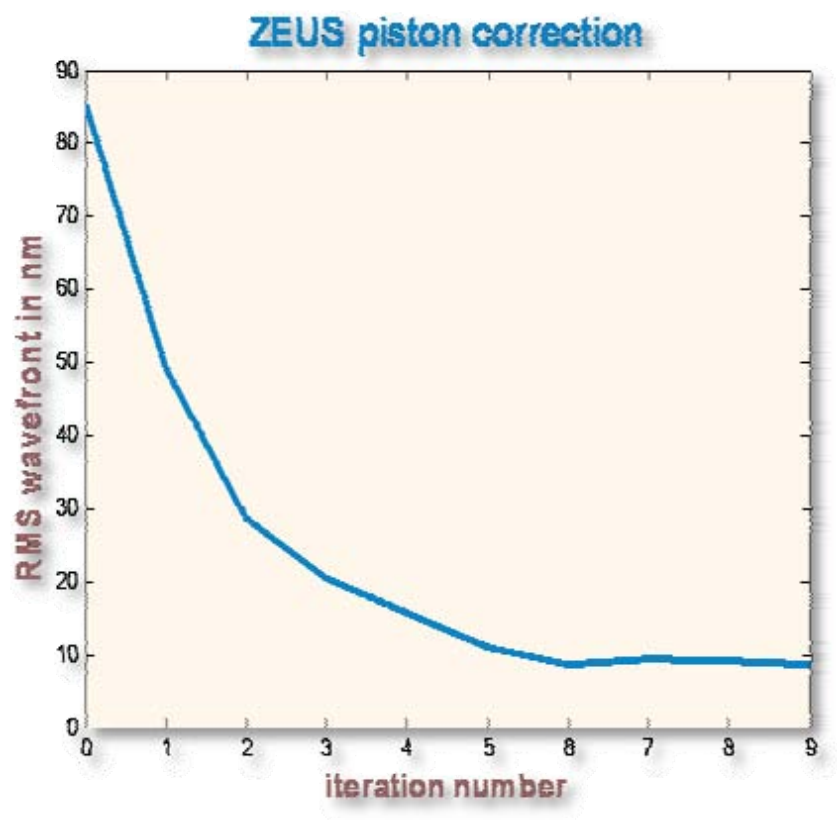

Fig. 4. Piston correction made by ZEUS within the mono- $\lambda$ regime (obtained on sky)

Results have been obtained first in laboratory using a turbulence generator and then verified on sky on Paranal. The on-sky environment with the varying conditions like, temperature, seeing, wind speed or light scattering (from the moon for example) was radically different from the laboratory and had a direct impact of the OPSs behaviours. After the commissioning of the experiment each sensor 
had a dedicated time to follow its test plan. Each OPS have been able to perform the phasing correction. Figure 4 shows for example the result obtained with ZEUS with a bright star in the mono- $\lambda$ regime. The corrected surface is better than $10 \mathrm{~nm}$ RMS. This is a typical results for the OPSs with seeing better than 1 arcsec. the limiting star magnitudes for which the closed loop corrections would still converge have been determined for each OPS. The convergence has been obtained with magnitudes between 14 and 18 depending on the sensor. The PYPS measurement is by far the best taking advantages of the few numbers of pixels used to detect the signal compare to the other OPSs. The loop converged with a final precision better than $25 \mathrm{~nm}$ rms. With the multi- $\lambda$ technique, most sensors were capable of extending the capture range typically to a few microns. DIPSI using a set of four optical filters. After seven iterations, the initial random wavefront piston error of $550 \mathrm{~nm}$ RMS was reduced to $30 \mathrm{~nm}$ RMS. PYPS, SHAPS and ZEUS were also able to close the loop for the correction of segment tip and tilt errors, achieving a final precision better than 0.4 arcsec rms. This is equivalent to a residual error at the borders of the segments of $16 \mathrm{~nm}$ wavefront rms.

\section{Conclusion}

The Active Phasing Experiment (APE) has been developed by ESO and its partners to gain experience in controlling segmented primary mirrors in preparation for the European Extremely Large Telescope. The experiment has tested various phasing techniques and has explored their advantages and limitations. The complexity of the phasing has been understood under seeing limited conditions. More work is still to be done to understand the behaviour in case of atmospheric correction using adaptive optics systems.

\section{Acknowledgement}

This experiment has been supported by the FP6 research programme of the European Union. The consortium which participated to this research project is composed of the Instituto de Astrofísica de Canarias in La Laguna in Tenerife in Spain, Fogale Nanotech in Nîmes in France, the Laboratoire d'Astrophysique de Marseille in France, the Osservatorio Astrofisico di Arcetri (INAF) in Italy and of the European Southern Observatory. I would like to thanks all the participants for their dedication to the project.

\section{References}

1. Wilhelm et al., Appl. Opt. 47, 29 (2008)

2. Montoya-Martinez L. et al. Proc. SPIE 6267 (2006)

3. Pinna et al., Proc. SPIE 7012 (2008)

4. Marchetti et al., ., Proc. SPIE 7015 (2008)

5. Mazzoleni et al., Proc. SPIE 7012 (2008)

6. Surdej et al., Proc. SPIE 7012 (2008)

7. Yaitskova et al., JOSA A 22, 6 (2005) 